\title{
DISTRIBUTED DYNAMIC FREQUENCY ALLOCATION IN WIRELESS CELLULAR NETWORKS USING GRAPH MULTICOLORING
}

\author{
Narayan Patra ${ }^{1}$, B N Bhramar Ray ${ }^{2}$, Shakti Prasad Mohanty ${ }^{3}$ \\ ${ }^{I}$ PhD Scholar, Dept of Comp Sc., Utkal University, Bhubaneswa, Odisha, India \\ ${ }^{2}$ Sr Lecturer, PhD Scholar, Dept of Comp Sc., Utkal University, Bhubaneswa, Odisha, India \\ ${ }^{3}$ Professor, Dept of Mathematics, College of Engineering and Technology, Bhubaneswa, Odisha, India
}

\begin{abstract}
Frequency Allocation Problem in wireless cellular networks is a major area of research in telecommunication industries. Since users of mobile phones are increasing exponentially, the existing limited frequency spectrum is unable to provide services efficiently. To enhance the performance of the networks, some measures must be taken to reduce drop call probabilities due to non availability of free frequency. The existing frequency allocation scheme call "hybrid with borrow" drops the calls regularly if frequencies are not free in a given cell. In this paper, we have developed a new scheme called "HYBORROW", which elegantly increases the utilization of new drop call probability by $20 \%$ over an existing scheme by borrowing the frequencies from neighbor cells.
\end{abstract}

Keywords: Drop call, Hybrid, borrow, multicoloring, cellular Network.

\section{INTRODUCTION}

In recent years, there has been a tremendous improvement in the field of wireless cellular networks. The cellular principle partitions a geographical area into cells which are of regular hexagons in shape due to V.H. MacDonald et al. [1]. Each cell has a base station (BS) and a number of mobile terminals e.g mobile phone. The base station is equipped with a transceiver operates on low power energy. Each BS monitors all the mobile units within its own cell. The BSs are connected to a central controller called Mobile Switching Center (MSC) through high speed wired links. To establish wireless connections among mobile phones it is essential to assign frequencies to them in such a way that no two calls originating from the same or neighboring cells are assigned by the same frequency. Frequency Division Multiplexing (FDM) technique is more often used for this purpose in cellular networks. Since we have limited frequencies in comparison to the number of users, it is necessary to utilize the given frequency spectrum optimally. Again the interference of frequencies is a measure issue in the frequency assignment problem. So during the process of frequency allocation, the network operators must take care of avoiding interference among the same frequencies. Basically there are three types frequency interferences take place in networks and they are known as co-site, adjacent and co-channel interferences. Hence to exploit the above limitations of frequency allocations, the following points must be taken care. (1) Span of frequencies must be minimized; (2) interference constraints should be respected and (3) the number of drop calls must be reduced.

Graph multicoloring is a well known alternative to solve frequency allocation problem in wireless cellular networks as studied by L. Narayanan [2]. Many researchers have proposed different techniques such as FAA, Greedy, Hybrid,
HYBRID_RE and Borrow based on both offline and online approaches as in [1, 3, 4, 5, and 6]. They have considered the frequency allocation problems by assuming call duration finite or infinite. The Fixed Assignment Algorithm (FAA) as suggested by V. MacDonald et al. in [1] is a simple non re-coloring strategy for frequency allocation. In this strategy, the nodes are partitioned into independent sets and each set is assigned exclusively by separate sets of frequencies. If the number of calls is uniformly distributed in the network, we can see this strategy works well. The greedy strategy implements to solve the problem with recoloring and non recoloring by Chan et al. [7]. Using this scheme, every call is assigned with minimum numbered free frequency that has not been allocated to any other call from same or adjacent cells to avoid interference. In Hybrid scheme, both FAA and greedy strategies are implemented in order to allocate frequencies efficiently. This scheme is also investigated using without frequency reassignment due to Chan et al. [4] and with frequency reassignment due to Patra et al. [5]. Borrow is another strategy to improve the performance of FAA by borrowing available frequencies from the neighbor cells due to Chan et al. in [6].

Though, all schemes discussed so far have same objective, in practical scenarios each one has some limitations. As we know cellular network is a model of regular hexagonal graph which is a 3-colorable graph as studied by Deo N. [8]. We use three base colors i.e. Red, Green and Blue for coloring all the vertices of a hexagonal graph such that each vertex colored with one of the three base colors and no two adjacent vertices are of the same color. Whenever, the number of calls exceeds the number of frequencies preallocated to a cell, in this case the required frequency is borrowed from the adjacent cells. Hence the dropping of calls due to lack of free frequency can be minimized by 
applying the concept of borrow. Since there is no restriction on the selection of neighbor cells to borrow free frequency, it motivates us to improve the existing Hybrid and Borrow strategies due to Chan et al. [4] and Janssen et al. [9] is by introducing a combined strategy called Hybrid with Restricted Borrow (HYBORROW). The principle of the proposed strategy is elaborated in forth coming section. In summary our contributions in this paper are cited below.

- We propose a new distributed dynamic frequency allocation algorithm (HYBORROW) for new call with infinite duration.

○ Using the proposed algorithm, each cell is allowed to borrow frequency only from the specified neighbors instead of from its arbitrary neighbors with greedy approaches.

- We have done a comparative study between the existing borrow strategy and the proposed borrow strategy.

- We have also shown that the drop call probability of our proposed algorithm is less than drop call probability due to existing strategy.

- We claim that the drop call probability for both strategies decrease with increase in the number of frequencies.

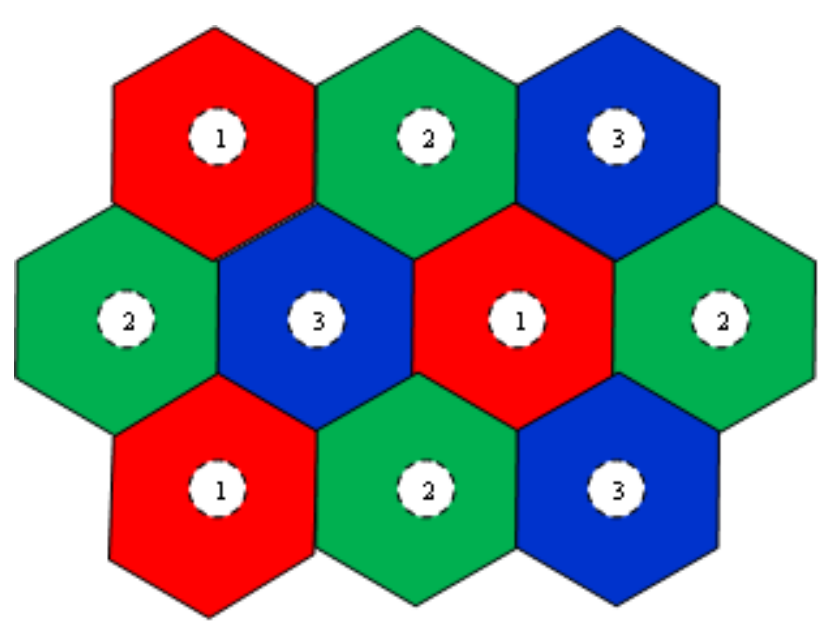

Fig. 1 3-Colorable hexagonal graph

The rest of this paper is organized as follows. In section II, we present HYBORROW a new algorithm for frequency allocation in cellular networks with restriction on borrows. The experimental results is work out in section III. Finally the conclusion and future scopes are drawn in section IV.

\section{PROPOSED NEW HYBRID WITH BORROW}

\section{ALGORITHM}

In this section, we introduce our new distributed frequency allocation algorithm for $\chi$-colorable network called HYBORROW. This algorithm implements combination of two methods as suggested by Chan et al. in [4] and [6]. Initially, the given set of frequencies is divided into $\chi+1$ number disjoint sets and each of the sets is allocated permanently to the cells depending on their base colors. Secondly, each call is assigned with minimum integer frequency using greedy approach. Finally, in case of shortage of frequency in a particular cell, it borrows additional frequencies dynamically from the specified sets. We know cellular networks are the models of 3-colorable hexagonal graphs. For a $\chi$-colorable network, the given integer frequency set $\mathrm{F}=\{1,2,3, \ldots\}$ is now divided into $\chi+1$ disjoint subsets denoted as $\mathrm{F}_{\mathrm{i}}$, for $\mathrm{i}=0,1,2 \ldots \chi$, according to the following rules due to Chan et al. in [4],

$$
F_{i}=\{f: f \equiv i \bmod (\chi+1)\}, \text { for } i=01,2 \ldots \chi \text {. }
$$

Thus for a particular case $\chi=3$, we have

$$
\begin{aligned}
& F_{0}=\{f: f \equiv 0 \bmod 4\}=\{4,8,12,16 \ldots\} \\
& F_{1}=\{f: f \equiv 1 \bmod 4\}=\{1,5,9,13, \ldots\} \\
& F_{2}=\{f: f \equiv 2 \bmod 4\}=\{2,6,10,14, \ldots\} \\
& F_{3}=\{f: f \equiv 3 \bmod 4\}=\{3,7,11,15, \ldots\}
\end{aligned}
$$

\subsection{Principles of HYBORROW Algorithm}

The proposed algorithm is executed by each of the base stations. It deals with a input sequence of requests $\sigma=\left\{\mathrm{c}_{x}^{j}\right\}$, where $x$ identifies base color of the cell such that $1 \leq x \leq \chi$, $\mathrm{j}=1,2, \ldots \mathrm{n}$, denotes request number and $\mathrm{c}$ represents the identity of the cell from which the call $\mathrm{c}_{x}^{j}$ is generated. Now assuming that call duration is infinite and each call is allocated with one frequency throughout its entire duration. On receiving the request, the base station starts executing the algorithm. Suppose a call arrives at a node $v$ with color $x(1 \leq x \leq 3)$. The call is assigned by the frequency according to following assignment scheme.

\subsection{Frequency Allocation Scheme}

For each request $\mathrm{c}_{x}^{j}$, supposing that it originates from a node $v$ with color $x(1 \leq x \leq 3)$, then assign a frequency from $\mathrm{F}_{x}$ to call according to following rules.

- Let $s$ be the minimum number of $\mathrm{F}_{x}$ such that frequency $s$ is not assigned to any call from $v$. Then assign frequency $s$ to the new call.

- If $\left|\mathrm{F}_{x}\right|=0$, then implement algorithm BORROW.

- Using BORROW, select the minimum number $t$ from the union of $\mathrm{F}_{x-1}, \mathrm{~F}_{x-2} \ldots \mathrm{F}_{1}$ and $\mathrm{F}_{0}$, where $x$ $(1 \leq x \leq 3)$ such that frequency $t$ is not allocated to any call from $v$ or its neighbors.

\section{Algorithm: HYBORROW}

Input: Sequence of requests $\left\{\mathrm{C}_{x}^{j}\right\}$, for $x=1$ to 3 and $\mathrm{j}=$ $1,2 \ldots \mathrm{n}$.

1. if $x=1$ then // cell with base color red

2. while $\left|\mathrm{F}_{x}\right| \neq 0$ then

3. $s=\operatorname{MIN}\left(\mathrm{F}_{x}\right)$

4. $\mathrm{f}_{\min } \leftarrow s$ 
5. if $\mathrm{f}_{\min }$ is not allocated to any call $\mathrm{C}_{x}^{j}$ in $\mathrm{C}_{x}$ then

6. $\mathrm{f}_{\mathrm{c}}=\mathrm{f}_{\min } / / / \mathrm{f}_{\mathrm{c}}$ is frequency for requested call

7. $\mathrm{F}_{x}=\mathrm{F}_{x^{-}}\left\{\mathrm{f}_{\mathrm{c}}\right\}$

8. end

9. end

10. BORROW( )

11. else if $x=2$ then //cell with base color green

12. while $\left|\mathrm{F}_{x}\right| \neq 0$ then

13. $s=\operatorname{MIN}\left(\mathrm{F}_{x}\right)$

14. $\mathrm{f}_{\min } \leftarrow s$

15. if $\mathrm{f}_{\min }$ is not allocated to any call $\mathrm{C}_{x}^{j}$ in $\mathrm{C}_{x}$ then

16. $\mathrm{f}_{\mathrm{c}}=\mathrm{f}_{\min } / / \mathrm{f}_{\mathrm{c}}$ is frequency for requested call

17. $\mathrm{F}_{x}=\mathrm{F}_{x}-\left\{\mathrm{f}_{\mathrm{c}}\right\}$

18. end

19. end

20. BORROW( )

21. else //cell with base color blue

22. while $\left|\mathrm{F}_{x}\right| \neq 0$ then

23. $s=\operatorname{MIN}\left(\mathrm{F}_{x}\right)$

24. $\mathrm{f}_{\min } \leftarrow s$

25. if $\mathrm{f}_{\mathrm{min}}$ is not allocated to any call $\mathrm{C}_{x}^{j}$ in $\mathrm{C}_{x}$ then

26. $\mathrm{f}_{\mathrm{c}} \leftarrow \mathrm{f}_{\min } / / \mathrm{f}_{\mathrm{c}}$ is frequency for requested call

27. $\mathrm{F}_{x} \leftarrow \mathrm{F}_{x}-\left\{\mathrm{f}_{\mathrm{c}}\right\}$

28. end

29. end

30. BORROW( )

31. end

\subsection{Principles of Borrow Algorithm}

The disadvantages due to fixed frequency allocation scheme were avoided by allowing a cell to borrow free frequencies from its neighbors such that no two frequencies interfere. In this way a cell was able to fulfill its requirements for frequencies on demand. There was also no restriction on borrow the required frequencies from the specific neighbors. However, in our proposed algorithm, a cell borrows the shortage of frequencies from its neighbors including from a sharable frequency set $F_{0}$ which is available at MSC. Again there is also restriction on this scheme such that a cell with base color $x$ can borrow from a cell whose base color is less than $x$, where $1 \leq x \leq 3$. It selects minimum valued free frequency using greedy approach. The details procedure is given in the algorithm BORROW.

\section{Algorithm: BORROW}

Input: Base color $x$ associated with a call request $\mathrm{C}_{x}^{j}$.

1. Broadcast request for $\mathrm{F}_{0}$ from $\mathrm{MSC}$

2. if $x=1$ then //cell with base color Red

3. $t=\operatorname{MIN}\left(\bigcup_{i=0}^{x-1} \mathrm{~F}_{\mathrm{i}}\right)$

4. $\quad \mathrm{f}_{\min } \leftarrow t$

5. if $\mathrm{f}_{\min }$ is not allocated to any call in $v$ or its neighbor then

6. $\quad \mathrm{f}_{\mathrm{b}} \leftarrow \mathrm{f}_{\min } / / \mathrm{f}_{\mathrm{b}}$ is borrowed for requested call

7. end

8. else if $x=2$ then //cell with base color Green
9. $t=\operatorname{MIN}\left(\bigcup_{i=0}^{x-1} \mathrm{~F}_{\mathrm{i}}\right)$
10. $\mathrm{f}_{\min } \leftarrow t \quad \mathrm{f}_{\min }$ is not allocated to any call in $v$ or its
11. neighbor then

12. $\mathrm{f}_{\mathrm{b}} \leftarrow \mathrm{f}_{\min } / / \mathrm{f}_{\mathrm{b}}$ is borrowed for requested call

13. end

14. else //cell with base color Blue

15. $t=\operatorname{MIN}\left(\bigcup_{i=0}^{x-1} \mathrm{~F}_{\mathrm{i}}\right)$

16. $\mathrm{f}_{\min } \leftarrow t$

17. if $\mathrm{f}_{\min }$ is not allocated to any call in $v$ or its neighbor then

18. $\mathrm{f}_{\mathrm{b}} \leftarrow \mathrm{f}_{\min } / / \mathrm{f}_{\mathrm{b}}$ is borrowed for requested call

19. end

20. end

\section{EXPERIMENTAL RESULTS}

In this section, we describe our new hybrid with borrow frequency allocation algorithm for a 3-colorable hexagonal graph $G=(V, E)$, where $V$ is set of vertices and $E$ is set of edges. Each of the vertices corresponds to base station of a cell and each edge corresponds to link between base station and mobile units. As we know a regular hexagonal graph is 3-colorable, using three base colors Red, Green and Blue, each of cells is identified by its id number and color. A set of finite integer frequencies is provided to the network operator to allocate all the calls that are generated in the networks.

Let us suppose the number frequencies are divided into four equivalence classes with same size as discussed in the previous section. From the figure 1 consider any three cells which are mutually adjacent to each other. Now applying the existing hybrid and the proposed hybrid with borrow strategies for frequency allocation problem, we investigate the properties of drop call probabilities.

\subsection{Using existing Hybrid with Borrow strategy}

Let us suppose the number of calls is uniformly distributed over all the cells. Consider three cells $\mathrm{m}, \mathrm{n}$ and $\mathrm{o}$, which form a clique $\mathrm{K}$. Using the existing strategy of hybrid with borrow, any cell in $\mathrm{K}$ can allocate maximum of $3 \mathrm{M}=$ $\left|\left(\bigcup_{i=1}^{x} F_{i}\right)\right|$, where $\left|F_{i}\right|=M$ ( number of frequencies available for allocation), for $\mathrm{i}=1,2,3$. Since we are dealing with infinite call duration, according to above strategy, if there will be any further calls generated in any one of the cells after allocating $3 \mathrm{M}$ calls, the requested call will be dropped. Now we will calculate the probability of drop calls $\left(\mathrm{P}_{\mathrm{D}}\right)$ in any cell belongs to $\mathrm{K}$ in this scenario. Let $\mathrm{DC}$ be number of drop calls is defined as the number of requested calls minus number of free frequencies available in three disjoint sets. 
Thus, $\mathrm{P}_{\mathrm{D}}=\frac{D C}{\text { number of requested calls }}=\frac{(3 M+1)-3 M}{3 M+1}$,

where $\mathrm{M}$ is maximum number frequencies available in each of the integer frequency sets $F_{i}, i=1,2,3$. Thus, probability of minimum number of drop call in each cell is given by,

$$
\mathrm{P}_{\mathrm{D}}=\frac{1}{3 M+1} .
$$

\subsection{Using proposed Hybrid with Borrow strategy (HYBORROW)}

In this strategy, the integer frequency set is divided into four equivalent classes known as $F_{i}$, for $i=0,1,2$ and 3 of same size, where $F_{0}$ is a sharable set. Calls from any cell irrespective of their base colors use $\mathrm{F}_{0}$ for allocation. Remaining sets $F_{i}$, for $i=1,2$ and 3 are dedicated for use only in the cells with corresponding base color $\mathrm{x}=1,2$, and 3 respectively. Now implementing our proposed algorithm, we will find the probability of minimum number of drop calls as follows.

Let us suppose cells $\mathrm{p}, \mathrm{q}$ and $\mathrm{r}$ form a clique in the given regular hexagon graph. In this case any cell with base color $x(1 \leq x \leq 3) \quad$ can allocate frequency to maximum $\left|\left(\bigcup_{i=0}^{x} \mathrm{~F}_{\mathrm{i}}\right)\right|$ calls, where $\mathrm{F}_{\mathrm{i}}$ is the set of free integer frequencies.

Thus, if $x=1$ (red color), the cell with color red can allocate maximum $\left|\mathrm{F}_{0} \cup \mathrm{F}_{1}\right|$ calls

if $\quad x=2$ (green color), the cell with color green can allocate maximum $\left|F_{0} \cup F_{1} \cup F_{2}\right|$ calls and if $x=3$ (blue color), the cell with blue color can allocate maximum $\mid$ $\mathrm{F}_{0} \cup \mathrm{F}_{1} \cup \mathrm{F}_{2} \cup \mathrm{F}_{3} \mid$ calls. Assume $\left|\mathrm{F}_{0}\right|=\left|\mathrm{F}_{1}\right|=\left|\mathrm{F}_{2}\right|=\left|\mathrm{F}_{3}\right|=$ $\mathrm{Y}$, where $\mathrm{Y}$ is number of distinct free frequencies available free frequencies available in each set. Now cell $\mathrm{p}$ with base color $x=1$, can allocate maximum $2 \mathrm{Y}$ calls; cell $\mathrm{q}$ with base color $x=2$, can allocate maximum $3 \mathrm{Y}$ calls and cell $\mathrm{r}$ with base color $x=3$, can allocate maximum 4Y calls. If a cell generates at least one more new call which is beyond its capacity for allocation of channels, then the excess calls will be dropped.

Therefore, $\quad \mathrm{P}_{D}^{\prime}$ (probability of drop calls in cell $\left.\mathrm{p}\right)=$ $\frac{1}{2 Y+1}$, where $2 \mathrm{Y}+1$ maximum number of calls generated in $\mathrm{p}, \mathrm{P}_{D}^{\prime}$ (probability of drop calls in cell $\left.\mathrm{q}\right)=\frac{1}{3 Y+1}$, where $3 \mathrm{Y}+1$ maximum number calls generated in $\mathrm{q}$ and $\mathrm{P}_{\mathrm{d}}$ $($ probability of drop calls in cell $\mathrm{r})=\frac{1}{4 Y+1}$, where $4 \mathrm{Y}+1$ maximum number calls generated in $\mathrm{r}$. Now taking the minimum of these probability values, we have
$\mathrm{P}_{D}^{\prime}=\frac{1}{4 Y+1}$.

From equations (1) and (2) it is concluded that probability of drop calls due to the proposed hybrid with borrow frequency allocation strategy is less than the probability of drop calls due to the existing hybrid with borrow frequency allocation strategy. Using Scilab 5.4.1 software we have investigated the graphs given in the figure 2 for the drop call probabilities by considering the two strategies. It is found that drop call probabilities for both cases decrease when the cardinality of the integer frequency sets increase.

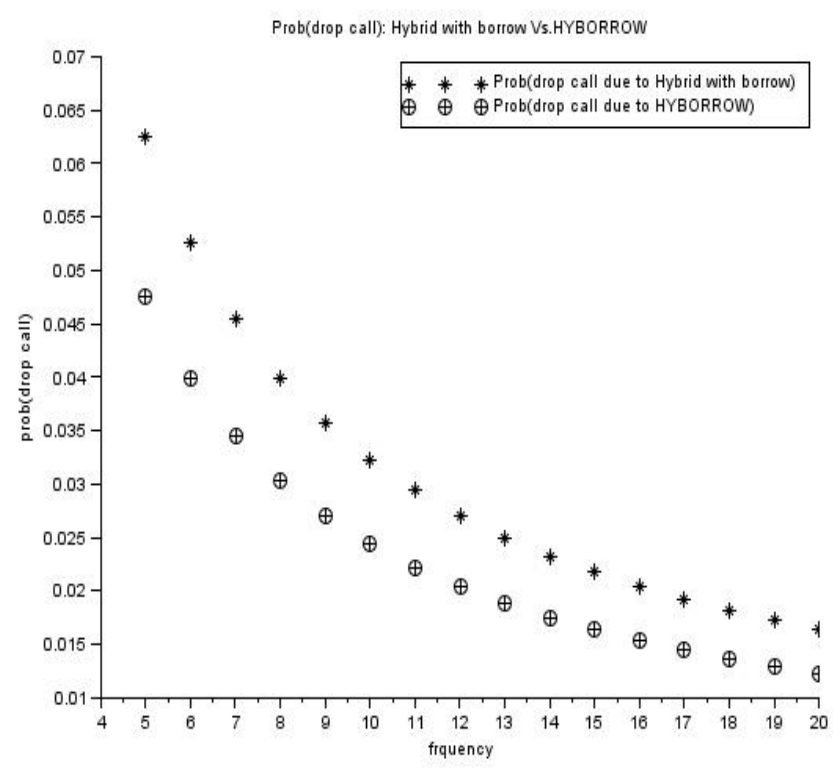

Fig.-2 Drop call probabilities vs. frequency frequencies

\section{CONCLUSION}

One of the characteristics of wireless cellular networks, which badly impacts on its performance is call drop due to non availability of free frequencies. We reduced it through our new hybrid scheme in addition to frequency borrowing facility. By comparing the drop call probability due to earlier method of hybrid with borrowing frequency, we verified that our scheme reduced $20 \%$ of the earlier one. When the availability of frequencies increases, it is also verified that the drop call probability proportionately reduces.

\section{REFERENCES}

[1]. MacDonald. "Advanced mobile phone service: The cellular concept." Bell Systems Technical Journal, 1979.

[2]. L. Narayan, "Channel assignment and graph multicoloring", Handbook of wireless networks and mobile computing, pages 71-94. John Willey \& Sons.

[3]. I. Caragiannis, C. Kaklamanis, and E. Papaioannou. "Efficient on-line frequency assignment and call control in cellular networks." Theory Comput. Systems, 2002. 
[4]. J. W.T. Chan, F. Y. L. Chin, D.Ye. and Y. Zhang, "Online frequency assignment in cellular networks." In Proc.19the Symp. On parallel Algorithms and Architectures (SPAA), 2007.

[5]. Patra Narayan, Bhramar Ray B. N. and Mohanty S. P, “ Online frequency reassignment for new and drop calls in wireless cellular networks", ICIT-2014.

[6]. J. W.-T. Chan, F. Y. L. Chin, D. Ye, Y. Zhang, and H. Zhu. "Frequency Assignment Problems for linear cellular networks." In Proceedings of the 17th Annunal International Symposium on Algorithms and Computation (ISAAC), pp. 61-70, 2006.

[7]. J. W.T. Chan, F. Y. L. Chin, D. Ye, Y. Zhang, and H. Zhu. "Greedy online frequency assignment in cellular networks." Inf. Process. Lett., 2007.

[8]. Deo N. ,"Graph Theory with applications to engineering and computer science", PHI, 2001.

[9]. J. Janssen, D. Krizanc, L. Narayanan, and S. M. Shende. Distributed online frequency assignment in cellular networks. J. Algorithms, 36(2):119\{151, 2000.

\section{BIOGRAPHIES}

Narayan Patra received his M.Sc degree in Mathematics from Utkal University,M.Tech in Computer Science from Utkal University. He has 15 years of teaching experience in the lleading engineering colleges under BPUT in Odisha, India. His research interest is in channel allocation problem in wireless cellular networks

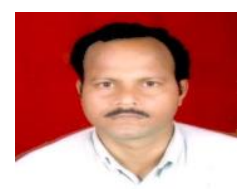

B N B Ray received his M.Sc in Mathematics from Utkal University, M.Tech in Computer Science from IIT Karaghpur and Ph. D degree from Utkal University. His research interests are in parallel algorithm, channel assignment in wireless network and VLSI. He is now working as Senior Lecturer in the PG Dept. of Computer Science and Application of Utkal University, Bhubaneswar, India

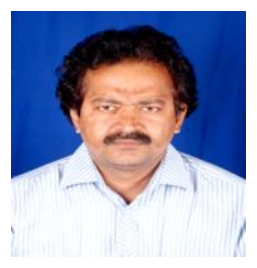

S P Mohanty received his M.Sc degree in Mathematics from Utkal University and $\mathrm{Ph} . \mathrm{D}$ degree from Utkal University. $\mathrm{He}$ is currently working as a Professor in the dept of Mathematics in the College of Engg.\& Technology, Bhubaneswar, India.His research interest is in parallel computing, operation research and graph theory. 